\title{
HYDRODYNAMIC INTERACTIONS OF RELATIVISTIC EXTRAGALACTIC JETS WITH DENSE CLOUDS
}

\author{
Eunwoo Chol and Paul J. Witta \\ Department of Physics and Astronomy, Georgia State University, Atlanta, GA; echoi@chara.gsu.edu, wiita@chara.gsu.edu \\ AND \\ Dongsu Ryu \\ Department of Astronomy and Space Science, Chungnam National University, Daejeon, Korea; ryu@canopus.cnu.ac.kr \\ Received 2006 August 9; accepted 2006 October 9
}

\begin{abstract}
We have studied three-dimensional hydrodynamic interactions of relativistic extragalactic jets with two-phase ambient media. These jets propagate through a denser homogeneous gas and then impact clouds with densities 100 to 1000 times higher than the initial beam density. The deflection angle of the jet is influenced more by the density contrast of the cloud than by the beam Mach number of the jet. A relativistic jet with low relativistic beam Mach number can eventually be slightly bent after it crosses the dense cloud; however, we have not seen permanently bent structures in the interaction of a high relativistic beam Mach number jet with a cloud. The relativistic jet impacts on dense clouds do not necessarily destroy the clouds completely, and much of the cloud body can survive as a coherent blob. This enhancement of cloud durability is partly due to the geometric influence of the off-axis collisions we consider and also arises from the lower rate of cloud fragmentation through the Kelvin-Helmholtz instability for relativistic jets. To compare our simulations with observed extragalactic radio jets, we have computed the approximate surface distributions of synchrotron emission at different viewing angles. These surface intensity maps show that relativistic jets interacting with clouds can produce synchrotron emission knots similar to structures observed in many VLBI-scale radio sources. We find that the synchrotron emission increases steeply at the moment of impact and the emission peaks right before the jet passes through the cloud.
\end{abstract}

Subject headings: galaxies: active — galaxies: jets — hydrodynamics — ISM: clouds — methods: numerical — relativity

\section{INTRODUCTION}

Relativistic jets emerging from extragalactic sources associated with active galactic nuclei (AGNs) are the most important means of transporting energy and mass from AGNs to an external medium over large distances. To understand how these relativistic jets interact with an inhomogeneous external medium containing small, dense gas clouds or clumps has been recognized as important for a long time. These interactions may substantially change the direction of relativistic jet flows, trigger extensive star formation in the shocked clouds, and possibly explain the basic mechanism behind the morphology of many extragalactic radio jets (Fanaroff \& Riley 1974).

Recent observations have revealed strong evidence of features associated with changes in jet directions resulting from interactions with small gas clouds in the narrow-line regions of Seyfert galaxies (e.g., Mundell et al. 2003). Fast outflows of gas observed in the central regions of powerful radio galaxies can also be caused by such interactions (e.g., Emonts et al. 2005; Morganti et al. 2005). The most likely interpretation of fast outflows is that all gas clouds are not destroyed by the jet; some clouds can severely disrupt the jet while other clouds are accelerated to the observed high outflow velocities by the thrust of the jet. It has been argued that in spite of the high energies involved in the interactions, only a few percent of the outflowing gas appears to be ionized, while the rest of the gas cools and becomes neutral due to highly efficient cooling near the jet bow shock.

Previous numerical works have used nonrelativistic hydrodynamic simulations to investigate jet interactions with clouds (de Gouveia Dal Pino 1999; Higgins et al. 1999; Wang et al. 2000; Saxton et al. 2005) or jets crossing a medium interface (e.g., Wiita et al. 1990; Wiita \& Norman 1992), focusing on the effects of the interactions on the morphology and kinematics of jets. Others have studied shock interactions, focusing on the structure and evolution of the clouds produced by the interactions in adiabatic cases (Klein et al. 1994; Xu \& Stone 1995; Poludnenko et al. 2002) and in radiative cases (Mellema et al. 2002; Fragile et al. 2004). According to de Gouveia Dal Pino (1999), simulations with conditions appropriate to protostellar jets making off-axis collisions with clouds produced a deflected beam. The deflection angle tended to decrease with time as the beam slowly penetrated the cloud, and when the jet penetrated most of the cloud, the deflected beam faded and the jet resumed its original propagation direction. Wang et al. (2000) found that powerful extragalactic jets eventually destroyed the clouds they considered, and these collisions induced nonaxisymmetric instabilities in the jets; weak jets can be effectively halted or destroyed by massive clouds; and slow, dense jets that were bent remained stable for extended times. Synthetic radio images produced by hydrodynamic simulations for comparison with observations also supported the hypothesis that these interactions are responsible for the distorted structures of some radio jets (e.g., Higgins et al. 1999). All those numerical works considered nonrelativistic jet speeds less than $0.5 c$, but the observed apparent superluminal motions of extragalactic radio sources indicate intrinsic jet speeds up to at least $0.98 \mathrm{c}$ (Zensus 1997). Thus, it is essential to perform relativistic hydrodynamic simulations of this problem in order to cover the range of true jet speeds.

Since time-dependent numerical simulations of relativistic jets were first reported (van Putten 1993; Duncan \& Hughes 1994; Martí et al. 1994), multidimensional relativistic hydrodynamic simulations have been used as an important method in understanding 
relativistic jets (Martí et al. 1997; Komissarov \& Falle 1998; Aloy et al. 1999; Rosen et al. 1999; Hughes et al. 2002; Mizuta et al. 2004). The morphological and dynamical properties of relativistic jets propagating through a homogeneous medium were studied by Martí et al. (1997) in two dimensions and by Aloy et al. (1999) in three dimensions. Komissarov \& Falle (1998) investigated the large-scale flows produced by classical and relativistic jets in a uniform external medium using analytical and numerical studies. Hughes et al. (2002) performed, in three dimensions, a study of the deflection of relativistic jets by an oblique density gradient and of the precession of relativistic jets. They found that fast relativistic jets can be significantly influenced by an oblique density gradient, showing a rotation of the Mach disk with the flow bent via a strong oblique internal shock.

In this paper we present results from three-dimensional hydrodynamic simulations of the interactions of relativistic jets with dense clouds. We focus on the off-axis collision of the relativistic jet with a steady spherical cloud. The main concerns of this study are how the relativistic jets are influenced by these interactions and how the interaction affects the evolution of the cloud. In $\S 2$ we briefly outline the dynamical problem, while the basic equations, numerical method, and setup we employ are described in $\S 3$. In $\S 4$ we describe the results, and we present a summary and discussion in $\S 5$.

\section{PROBLEM DESCRIPTION}

We consider the three-dimensional interactions of relativistic jets with two-phase ambient media. These jets propagate through a denser ambient gas and then hit spherical clouds with densities higher than that of the ambient gas. The initial ratio of the cloud density, $\rho_{c}$, to the ambient medium density, $\rho_{a}$, and that of the beam density, $\rho_{b}$, to the ambient medium density, are respectively defined as

$$
\chi \equiv \frac{\rho_{c}}{\rho_{a}}, \quad \eta \equiv \frac{\rho_{b}}{\rho_{a}} .
$$

If we neglect complicating effects, including radiative cooling and gravity, and consider only hydrodynamic effects, then this problem can be relatively simple and depends only on a few hydrodynamic variables: the Mach number of the jet and the initial density contrasts given in equation (1). Any geometric effects, such as different impact zone sizes or cloud shapes, certainly will make differences in the evolutions of jets and clouds, but the overall dynamical evolutions should not be very sensitive to them. Thus, we focus on the evolutions of jets and clouds influenced by the above hydrodynamic effects.

The approximate propagation velocity of the jet through the homogeneous ambient medium can be obtained by the conservation of the momentum flux of the beam and ambient medium in the reference frame of the Mach disk (Martí et al. 1997). Assuming pressure equilibrium between the beam and the ambient medium, the conservation of the momentum flux is $\rho_{b} h_{b} \Gamma_{b}^{\prime 2} v_{b}^{\prime 2}=$ $\rho_{a} h_{a} \Gamma_{a}^{\prime 2} v_{a}^{\prime 2}$, with the relations $v_{b}^{\prime}=\left(v_{b}-v_{a}\right) /\left(1-v_{b} v_{a}\right), \Gamma_{b}^{\prime}=$ $\Gamma_{b} \Gamma_{a}\left(1-v_{b} v_{a}\right), v_{a}^{\prime}=-v_{a}$, and $\Gamma_{a}^{\prime}=\Gamma_{a}$. Here, $h$ is the specific enthalpy and $v^{\prime}$ and $\Gamma^{\prime}$ represent, respectively, velocity and Lorentz factor measured in the reference frame of the Mach disk, while $v$ and $\Gamma$ indicate those measured in the rest frame of the ambient medium. The subscripts $b$ and $a$ stand for the beam and the ambient medium, respectively. After substituting for the primed variables in terms of the unprimed ones, the conservation of the momentum flux is derived to be

$$
\rho_{b} h_{b} \Gamma_{b}^{2}\left(v_{b}-v_{a}\right)^{2}=\rho_{a} h_{a} v_{a}^{2} .
$$

Then the one-dimensional jet advance velocity, estimated in the rest frame of the ambient medium, is

$$
v_{a}=\frac{v_{b}}{\sqrt{1 / \eta^{*}}+1},
$$

where $\eta^{*}$ is given by

$$
\eta^{*}=\Gamma_{b}^{2} \frac{\rho_{b} h_{b}}{\rho_{a} h_{a}} .
$$

In the nonrelativistic limit ( $h \rightarrow 1, \Gamma \rightarrow 1), \eta^{*}$ approaches $\eta$, so that $v_{a}$ represents the classical jet advance velocity through the ambient medium, i.e., $v_{a}=v_{b} /\left[(1 / \eta)^{1 / 2}+1\right]$.

Based on this jet advance velocity, we define the dynamical timescale, called the beam crossing time,

$$
t_{\mathrm{bc}} \equiv \frac{2 r_{c}}{v_{a}}
$$

as the time taken for the beam to sweep a distance across the ambient medium equal to the diameter of a cloud with radius $r_{c}$. Since the timescale $t_{\mathrm{bc}}$ depends only on a single variable $v_{a}$ (for fixed cloud radius), it is extremely useful in comparing and characterizing the dynamical evolutions of both jets and clouds with different model parameters.

Although we use the beam crossing time as the primary timescale in this study, it is also interesting to estimate the cloud crushing and cooling timescales. The cloud crushing timescale is the time required for the beam to cross the cloud diameter during the phase of cloud compression, and if $v_{a}$ is nonrelativistic, this timescale can be approximated as $t_{\mathrm{cc}} \sim 2 \chi^{1 / 2} r_{c} / v_{a}$ (Klein et al. 1994). Clearly, $t_{\mathrm{bc}} \simeq t_{\mathrm{cc}}$ in the absence of clouds, and for dense clouds $(\chi \gg 1), t_{\mathrm{bc}}<t_{\mathrm{cc}}$. Following Fragile et al. (2004) the cloud cooling timescale can be roughly estimated from $t_{\text {cool }} \sim$ $C v_{a}^{3} /\left(\chi^{3 / 2} \rho_{c}\right)$, where the constant $C=7.0 \times 10^{-35} \mathrm{~g} \mathrm{~cm}^{-6} \mathrm{~s}^{4}$. With values reasonable for kiloparsec-scale extragalactic situations, $r_{c}=1 \mathrm{kpc}, v_{a}=0.1 c, \chi=100$, and $\rho_{c}=10^{2} m_{\mathrm{H}} \mathrm{cm}^{-3}$, we find that $t_{\mathrm{bc}}<t_{\mathrm{cc}} \sim t_{\text {cool }}$. Thus, cooling will not be extremely important during the cloud compression phase for the chosen values. For fixed density and cloud radius, the cloud cooling timescale becomes longer than the cloud crushing timescale as $v_{a}$ increases, so the effect of cooling is somewhat reduced for relativistic jets compared to nonrelativistic ones. For parameters more relevant to VLBI-scale jet/cloud collisions, $r_{c}=0.5 \mathrm{pc}, v_{a}=0.5 \mathrm{c}, \chi=10^{4}$, and $\rho_{c}=10^{6} m_{\mathrm{H} \mathrm{cm}} \mathrm{cm}^{-3}$, we have $t_{\mathrm{bc}} \sim t_{\text {cool }}<t_{\mathrm{cc}}$, so cooling would be more important in this case. A more detailed consideration of cooling timescales is beyond the scope of the current paper.

Three distinct evolutionary stages can be considered in this problem. There is an initial jet propagation stage where the jet advances through a homogeneous ambient medium with velocity $v_{a}$. Once a jet is launched, a bow shock propagates into the ambient medium; this is followed by a Mach disk shock in the beam that is quickly established during this stage. When the jet strikes the cloud, the jet transmits a shock into the cloud. If the cloud/ ambient density contrast is sufficiently large and the jet speed is relatively slow, the speed of the transmitted shock in the cloud is much slower than that of the bow shock of the jet. Thus, the bow shock entirely encloses the cloud, which leads to the development of the Kelvin-Helmholtz instability at the cloud surface (e.g., Klein et al. 1994). The final stage is when the jet passes through the cloud. In this phase the cloud begins to reexpand just after the jet reaches the rear edge of the cloud. At the same time, the jet propagates in the original direction or in a new direction. 


\section{NUMERICAL SIMULATIONS}

\subsection{Basic Equations}

The special relativistic hydrodynamic equations are written in a covariant form (e.g., Landau \& Lifshitz 1959; Wilson \& Mathews 2003)

$$
\begin{gathered}
\partial_{\alpha}\left(\rho U^{\alpha}\right)=0, \\
\partial_{\alpha} T^{\alpha \beta}=0,
\end{gathered}
$$

where the energy momentum tensor is given by

$$
T^{\alpha \beta}=(e+p) U^{\alpha} U^{\beta}+p g^{\alpha \beta},
$$

and where $\partial_{\alpha}=\partial / \partial x^{\alpha}$ is the covariant derivative with spacetime coordinates $x^{\alpha}=\left[t, x_{j}\right], U^{\alpha}=\left[\Gamma, \Gamma v_{j}\right]$ is the normalized $\left(U^{\alpha} U_{\alpha}=-1\right)$ four-velocity vector, and a metric tensor $g^{\alpha \beta}$ with a signature +2 is used. The mass density, velocity, internal plus mass energy density, and pressure in the local rest frame are denoted by $\rho, v_{j}, e$, and $p$, respectively. Greek indices (e.g., $\alpha, \beta$ ) denote the spacetime components, while Latin indices (e.g., $i, j$ ) indicate the spatial components, and the speed of light is set to unity $(c \equiv 1)$ throughout this paper.

For our numerical purposes, it is convenient to rewrite the covariant equations (6)-(8) in the index form, which gives a hyperbolic system of conservation equations

$$
\begin{gathered}
\frac{\partial D}{\partial t}+\frac{\partial}{\partial x_{j}}\left(D v_{j}\right)=0, \\
\frac{\partial M_{i}}{\partial t}+\frac{\partial}{\partial x_{j}}\left(M_{i} v_{j}+p \delta_{i j}\right)=0, \\
\frac{\partial E}{\partial t}+\frac{\partial}{\partial x_{j}}\left[(E+p) v_{j}\right]=0,
\end{gathered}
$$

where the equation of state (EOS) is given by

$$
p=(\gamma-1)(e-\rho)
$$

and where, $D, M_{i}$, and $E$ are the mass density, momentum density, and total energy density in the reference frame, respectively, and $\gamma$ is the adiabatic index. We note that we restrict ourselves to an ideal gas EOS in this study (cf. Ryu et al. 2006).

The quantities in the reference frame are related to those in the local rest frame via the transformations

$$
\begin{gathered}
D=\Gamma \rho, \\
M_{i}=\Gamma^{2}(e+p) v_{i}, \\
E=\Gamma^{2}(e+p)-p,
\end{gathered}
$$

where the Lorentz factor is given by

\begin{tabular}{|c|c|c|c|c|c|c|c|c|}
\hline Model & $\chi$ & $\eta$ & $v_{b}$ & $\Gamma_{b}$ & $\mathcal{M}_{b}^{\mathrm{N}}$ & $\mathcal{M}_{b}^{R}$ & $t_{\mathrm{bc}}$ & $t_{\text {end }}$ \\
\hline M1 ...................... & 10 & 0.1 & 0.9 & 2.29 & 2.92 & 6.36 & 4.86 & $4 t_{\mathrm{bc}}$ \\
\hline 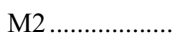 & 100 & 0.1 & 0.9 & 2.29 & 2.92 & 6.36 & 4.86 & $6 t_{\mathrm{bc}}$ \\
\hline M3 ..................... & 10 & 0.1 & 0.99 & 7.09 & 1.92 & 11.6 & 2.50 & $4 t_{\mathrm{bc}}$ \\
\hline M4 ..................... & 100 & 0.1 & 0.99 & 7.09 & 1.92 & 11.6 & 2.50 & $5 t_{\mathrm{bc}}$ \\
\hline
\end{tabular}

$$
\Gamma=\frac{1}{\sqrt{1-v^{2}}}
$$

with $v^{2}=v_{x}^{2}+v_{y}^{2}+v_{z}^{2}$.

If an EOS is assumed, the local sound speed $c_{s}$ and the specific enthalpy $h$ are easily derived. For an ideal gas, they are given by

$$
c_{s}^{2}=\frac{1}{h} \frac{\partial p}{\partial \rho}+\frac{\partial p}{\partial e}, \quad h=1+\frac{\gamma}{\gamma-1} \frac{p}{\rho} .
$$

TABLE 1

Simulation Parameters

Notes.-Here, $\chi$ is the ratio of the cloud density to the ambient medium density, $\eta$ is the ratio of the beam density to the ambient medium density, $v_{b}$ is the initial beam velocity, $\Gamma_{b}$ is the beam Lorentz factor, $\mathcal{M}_{b}^{\mathrm{N}}$ is the Newtonian beam Mach number, $\mathcal{M}_{b}^{R}$ is the relativistic beam Mach number, $t_{\mathrm{bc}}$ is the beam crossing time, and $t_{\text {end }}$ is the time at which the simulation is ended.

A $\gamma$-law gas such as an ideal gas has the local sound speed limit $c_{s} \leq(\gamma-1)^{1 / 2}$, and in the ultrarelativistic case $e \gg \rho$, the local sound speed approaches its limit [i.e., $c_{s} \rightarrow(\gamma-1)^{1 / 2}$ ].

\subsection{Numerical Method and Setup}

The system of equations (9)-(12) can be solved numerically with explicit finite difference upwind schemes that are based on exact or approximate Riemann solvers using the characteristic decomposition of relativistic hydrodynamic conservation equations. Although the upwind schemes were originally developed for nonrelativistic hydrodynamics, some schemes have been extended to special relativistic hydrodynamics while retaining the advantages of the upwind schemes, including high accuracy and robustness.

A multidimensional code for solving the special relativistic hydrodynamic equations as a hyperbolic system of conservation laws based on the total variation diminishing (TVD) scheme (Harten 1983) was developed and tested in Choi \& Ryu (2005). The TVD scheme is an explicit Eulerian finite difference upwind scheme and an extension of the Roe scheme to second-order accuracy in space and time. The code uses a new set of conserved quantities, which lead to a new eigenstructure for special relativistic hydrodynamics and employs an analytic formula for the calculation of the local rest-frame quantities from the referenceframe quantities. The advantage of our code is that it is simple and fast, and yet it is accurate and reliable enough. The performance of the code was demonstrated through several standard tests, including relativistic shock tubes, a relativistic wall shock, and a relativistic blast wave, as well as test simulations of the relativistic version of the Hawley-Zabusky shock and a relativistic extragalactic jet (Choi \& Ryu 2005). For our new simulations, we have parallelized this code using the message passing interface (MPI). The simulations described here typically use 64 processors on a Linux cluster.

Table 1 lists the initial parameters of the four different relativistic jet-cloud interaction models we have investigated in this study. All models use the adiabatic index $\gamma=4 / 3$ and assume pressure-matched jets and clouds, i.e., $p_{b} / p_{a}=p_{c} / p_{a}=1$, where $p_{b}, p_{c}$, and $p_{a}$ are the pressure of the beam, cloud, and ambient medium, respectively. We set $c=r_{c}=\rho_{a} \equiv 1$ in our models, so that all physical quantities are dimensionless and can be scaled to any specific physical model (e.g., $t \rightarrow t c / r_{c}, \rho \rightarrow \rho / \rho_{a}$ ). The Newtonian beam Mach number, $\mathcal{M}_{b}^{\mathrm{N}} \equiv v_{b} / c_{s, b}$, where $c_{s, b}$ is the sound speed in the beam, as well as the relativistic beam Mach number, $\mathcal{M}_{b}^{R} \equiv\left(\Gamma_{b} / \Gamma_{s, b}\right) \mathcal{M}_{b}^{\mathrm{N}}$, where $\Gamma_{s, b}$ is the Lorentz factor associated with the beam sound speed, are given in Table 1. As discussed in Königl (1980) in the context of relativistic gas dynamics, the relativistic Mach number is the best analog of the Newtonian one for nonrelativistic flows, so we use this relativistic 

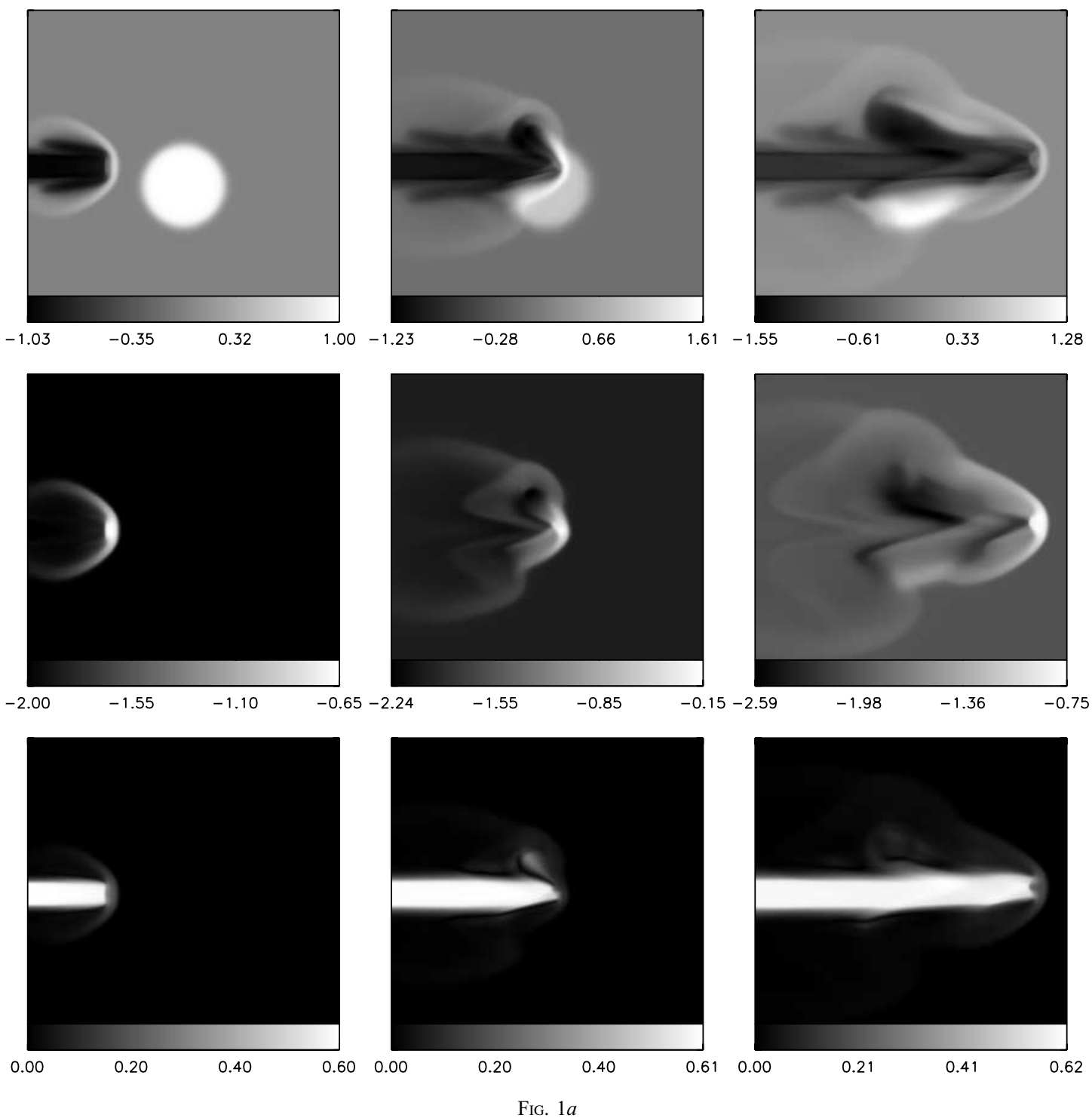

FIG. $1 a$

FIG. 1.- (a) Gray-scale images of density, pressure, and Lorentz factor (top to bottom) for model M1 at three different times, $t / t_{\mathrm{bc}}=1,2.5$, and 4 (left to right). The

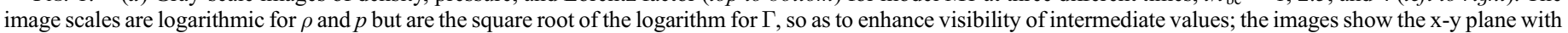

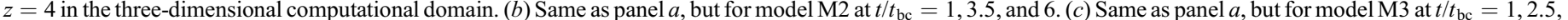
and 4. (d) Same as panel $a$, but for model M4 at $t / t_{\mathrm{bc}}=1,3$, and 5 .

beam Mach number to describe physical properties in our models. The initial density contrast between the beam and the ambient medium is fixed to $\eta=0.1$, so that jets strike clouds with densities 100 to 1000 times higher than the initial beam density. In models M1 and M2, the clouds interact with the lower relativistic beam Mach number jets, where the relativistic effects are less dominant, with smaller beam velocities and internal energies. Model M1 is almost identical to model M2 except for the smaller density ratio of the cloud to the ambient medium. Models M3 and M4 have been chosen to study the cloud interactions with the higher relativistic beam Mach number jets, with the more dominant relativistic effects caused by larger beam velocities and internal energies. Again, the initial conditions of model M3 are the same as those of model M4 except for the smaller density ratio of the cloud to the ambient medium.

We set up the density gradient of the spherical cloud edge with a hyperbolic tangent function

$$
\rho(r)=\frac{\rho_{c}+\rho_{a}}{2}+\frac{\rho_{c}-\rho_{a}}{2} \tanh \left(\frac{r_{c}-r}{\Delta r}\right),
$$

where $r$ is the distance from the center of the cloud and $\Delta r$ is the scale parameter for the width of density transition $\left(\Delta r \ll r_{c}\right)$. The presence of a true density discontinuity instead of this steep function would not affect the dynamics of jet-cloud interactions significantly, but the discontinuous cloud edge is approximated by this somewhat smoothed density profile to avoid numerical artifacts as the jet impacts the cloud. We assume that other physical quantities such as pressure and velocity are constant across the transition width.

The simulations have been performed in the three-dimensional computational domain with $x=[0,8], y=[0,8]$, and $z=[0,8]$ using a uniform Cartesian grid of $256^{3}$ cells. The beam, with a circular cross section of radius $r_{b}=1 / 4$ (8 cells), is initially located at $(x, y, z)=(0,4,4)$ and propagates through the ambient medium along the positive $x$-direction. In order for the relativistic jet to collide off-axis with the cloud at rest, the center of the cloud, with radius $r_{c}=1$ (32 cells), is placed at $(x, y, z)=(4,3.5,4)$; hence, the relativistic jet hits the spherical cloud with an impact angle of $30^{\circ}$. The outflow boundary condition is imposed on all boundaries of the computational domain except where the inflow 

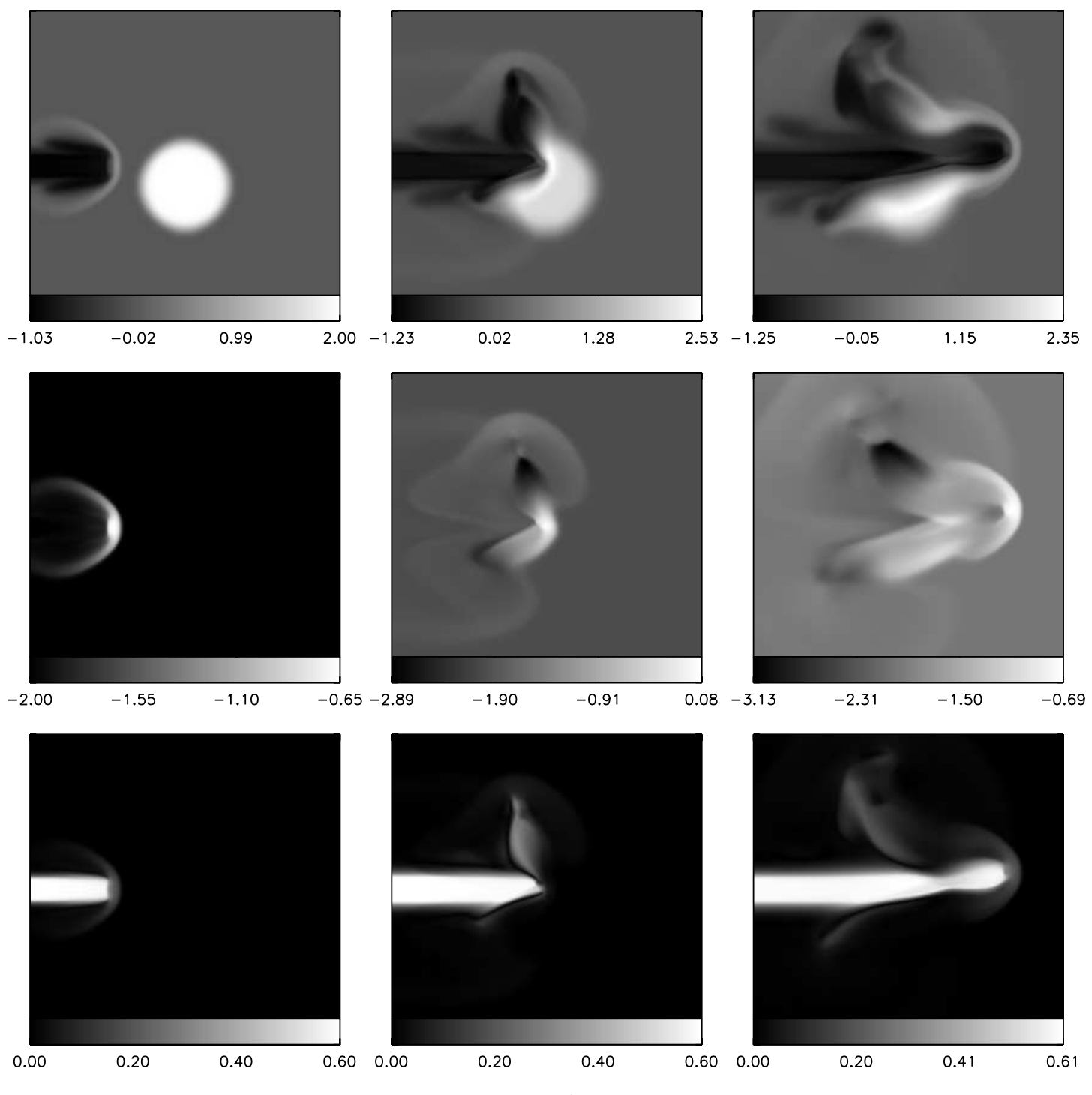

Fig. $1 b$

boundary condition is used to maintain the continuous jet. We were able to assign the relativistic jet only 8 cells per initial beam radius and the cloud 32 cells per initial cloud radius due to the limitation of computational resources. This resolution is less than that of previously reported two-dimensional works that are related to this problem. Thus, our three-dimensional simulations may not be fully converged, and some quantities to be described may change if three-dimensional simulations with much higher resolutions are performed in future studies; however, our tests of the code do indicate that simulations with this level of resolution should be reasonably accurate (Choi \& Ryu 2005).

\section{RESULTS}

\subsection{Morphology and Dynamics}

The gray-scale images in Figure 1 show the distinct evolutionary phases of models M1-M4, respectively. These images show the $x-y$ plane with $z=4$ in the three-dimensional computational domain. In each of these figures the top to bottom panels represent density, pressure, and Lorentz factor, respectively (in logarithmic scales), while the left to right panels represent evolutionary stages shown at three different times, $t=t_{\mathrm{bc}},\left(t_{\mathrm{bc}}+t_{\mathrm{end}}\right) / 2$, and $t_{\mathrm{end}}$.

The early stages of the relativistic jet propagation through the uniform ambient medium until the jet is about to collide the cloud $\left(t / t_{\mathrm{bc}} \sim 1\right)$ are basically similar to those found in earlier simulations (e.g., Martí et al. 1997; Aloy et al. 1999). Several key features are clear from the left panels of Figure 1. In all the models a bow shock that separates the jet from the external medium is driven, the beam itself is terminated by a Mach disk (terminal shock) where the beam kinetic energy is converted into its internal energy, and shocked jet material flows backward along the contact discontinuity (working surface) into a cocoon. There is no difference between models M1 and M2 and between models M3 and M4 at this stage because these two pairs of simulations have the same initial conditions of the jets and the same ambient media properties.

The relativistic beam Mach number of the jet is associated with the shape of the bow shock. In models M1 and M2, the lower relativistic beam Mach number jets, with a lower propagation velocity $\left(v_{a} \sim 0.42\right)$ and internal energy, have bow shocks with narrower conical shapes, and the Mach disk is quite close to the bow shock. This conical shape of the bow shock tends to be broader as the relativistic beam Mach number of the jet increases, as seen for models M3 and M4; these higher relativistic beam Mach number jets, with a higher propagation velocity $\left(v_{a} \sim 0.78\right)$ and internal energy, also have the Mach disk standing off farther from the bow shock. The shapes of the bow shocks are also connected with the sizes of the impact cross section when the jets begin to interact 

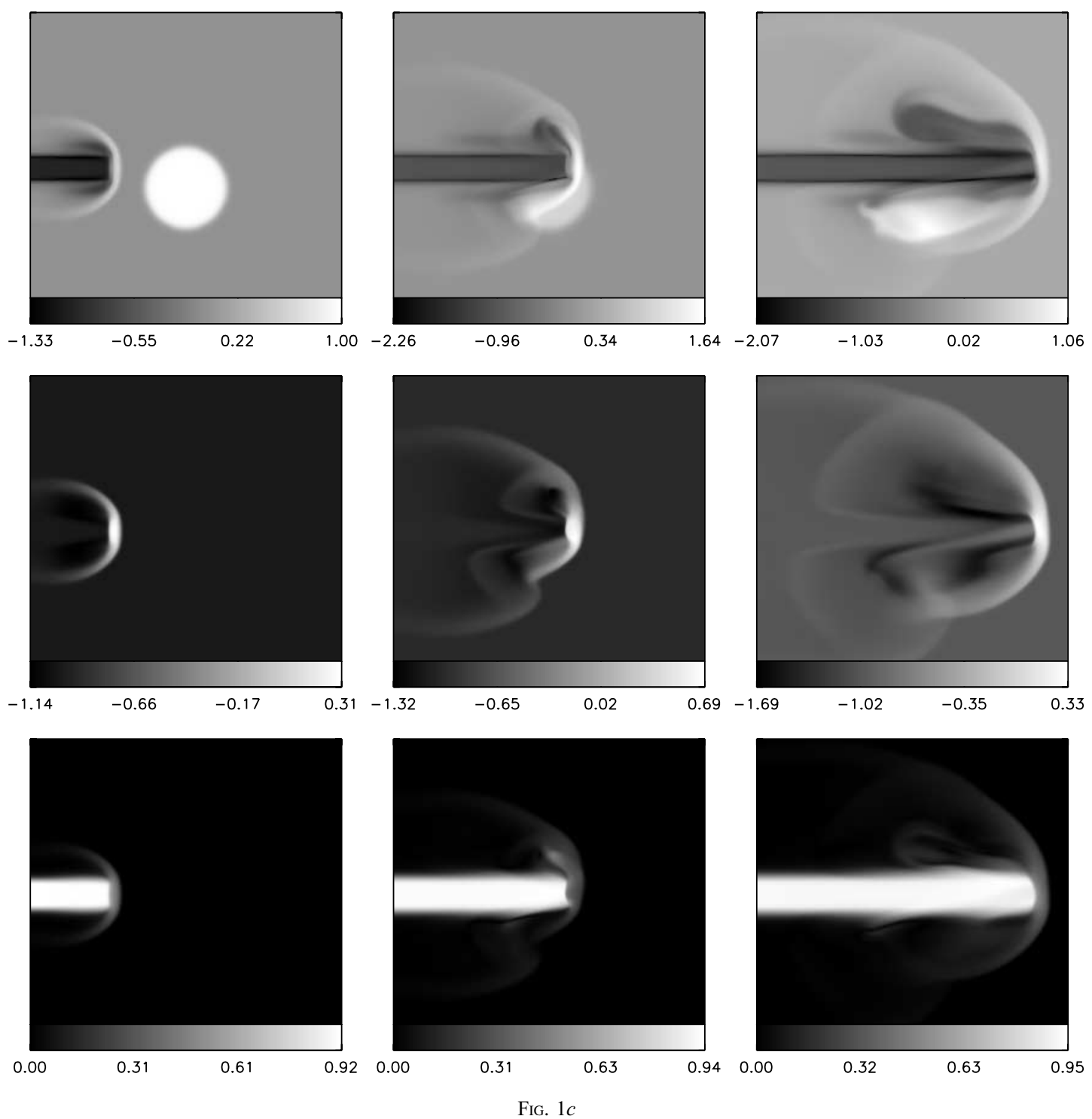

Fig. $1 c$

with the cloud. The low relativistic beam Mach number jets in models M1 and M2 feature relatively thick cocoons, while the high relativistic beam Mach number jets in models M3 and M4 have thin cocoons. This dependence of the cocoon morphology on the relativistic beam Mach number is consistent with previous results (see, e.g., Martí et al. 1997). Although the structural differences in the jet head and the cocoon are evident by this early stage of the evolution, the internal structures within the beam and backflows are not dominant and are barely distinguishable at this stage.

In every model, the relativistic jet begins to partially deflect as a direct response to its interactions with the clouds. This is seen in the middle column of panels of Figure 1; seen most clearly in the middle bottom panels are fast streams coming from the Mach disk at significant angles with respect to the jet axis. These deflection features are stronger in models M2 and M4, which have higher ratio of the density of the cloud to that of the ambient medium $(\chi=100)$. The deflection angles of the portion of the postMach shock flows with respect to the beam propagation axis are very time-dependent. In our models these angles peak when the jets cross over approximately half the clouds (at $t / t_{\mathrm{bc}} \sim 2.5,3.5$, 2.5, and 3 for models M1-M4, respectively). For the comparable dynamical times, models M2 and M4, both with $\chi=100$ but having different beam Mach numbers, show $80^{\circ}-90^{\circ}$ deflection angles, while models M1 and M3 with the same beam Mach numbers as the models M2 and M4, respectively, but with $\chi=10$, show smaller deflection angles of about $45^{\circ}$. This indicates that the deflection angle is more strongly influenced by the density contrast, $\chi$, than by the beam Mach number of the jet. For an offaxis collision there are weak deflection features on the other side of the jet axis, where the deflection of the outflow from the beam is significantly suppressed by the dense cloud. That suppression leads to the production of a strong oblique shock within the beam. As seen in the figures, the oblique shocks are quite strong in models M2 and M4, but in models M1 and M3 there are only relatively weak oblique shocks in the beam. Comparing at this stage models M1 and M3 with models M2 and M4, we note that the bow shocks enclose less of the cloud in models M1 and M3 because of their lower density contrast, $\chi$. That implies quicker penetration of the clouds by these jets, so the strengths of the oblique shocks in these beams are reduced.

Some additional properties of the simulations at this stage are shown in Figures 2 and 3. Figure 2 illustrates one-dimensional flow structures of density, pressure, and Lorentz factor along the beam propagation axis for models M1 and M3 at the same epoch as in Figures $1 a$ and $1 c$. In both models there are spikes in the 

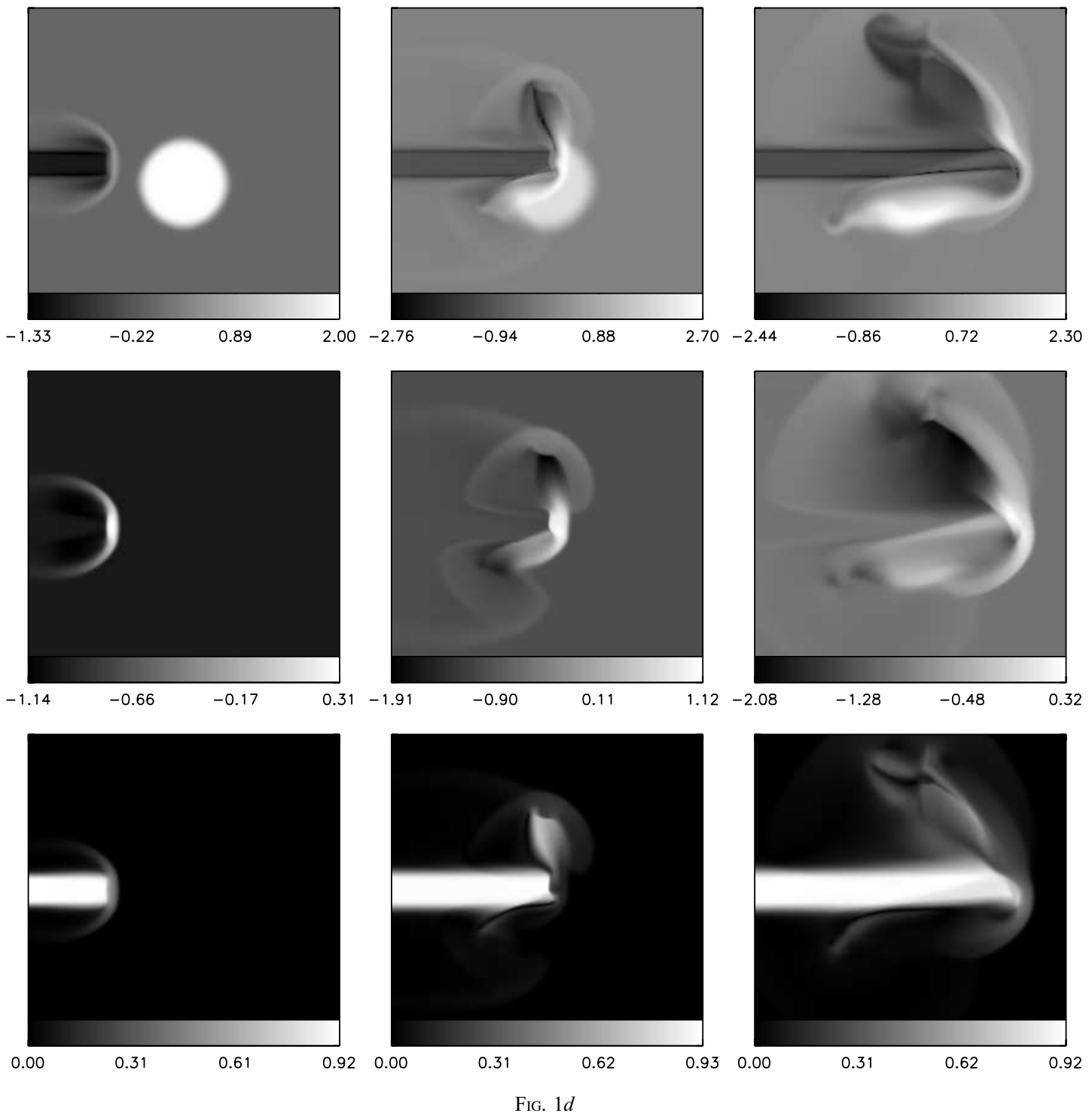

FIG. $1 d$

density and pressure associated with the impact by the incident jets, while there is little change in beam Lorentz factor. Figure 3 shows the images of the logarithm of the Lorentz factor projected at the viewing angle of $0^{\circ}$ for models $\mathrm{M} 2$ and $\mathrm{M} 4$, at $t / t_{\mathrm{bc}}=3.5$ and 3 , respectively. These projection images clearly show the anisotropic distribution and directions of the deflected gas induced by the jet. This gas is an admixture of jet and cloud material, but only a small fraction of the cloud gas is shown in these projection images, since the mean cloud velocity computed in each component (refer to $\S 4.2$ ) is $\left\langle v_{i}\right\rangle \lesssim 0.01$ and 0.06 for models M2 and M4, respectively, at the same epoch as in Figure 3. This implies that this deflected gas consists predominantly of jet material, although a small amount of cloud material is entrained in these deflected structures. This presence of deflected gas accelerating toward a terminal velocity strongly suggests that such deflected and accelerated gas is responsible for at least some of the outflowing gas observed in the vicinity of AGNs.

Once the jet passes through the cloud, it begins to accelerate, causing a change in the shape of the bow shock. As visible in the right panels of Figure 1, shown when the jet head nearly reaches the boundary of the computational cube (at $t / t_{\mathrm{bc}}=4,6,4$, and 5 for models M1-M4, respectively), the shape of the bow shocks changes more clearly in the low relativistic beam Mach number jets than in the high relativistic beam Mach number jets. That reflects the fact that the acceleration of the jets is somewhat faster in low relativistic beam Mach number jets. That reacceleration occurs in essentially the original propagation direction or in a somewhat new direction. In our simulations there is a trend for the flow of the jet to be bent more when a lower relativistic beam Mach number jet interacts with a denser cloud, with the least bending seen for model M3 and the most for model M2. We see in the right panels of Figure $1 b$ that the beam is bent by about $10^{\circ}$ with respect to the original jet axis. The bent jet still remains stable and collimated over the several dynamical times we could follow its development.

After the jet head passes the cloud, the amount of strongly deflected gas gradually reduces and the oblique shocks continue to develop in the beam. These oblique shocks are unlikely to play a major role in slowing the jets, because we do not find any significant deceleration features during this stage. Although a significant portion of the momentum flux of the jets is transferred to the deflected gas and the cloud through the collision events, the jets in our simulations are still stable and well collimated over several dynamical times after collisions, even if the jet is bent. This stable, collimated condition is quantitatively apparent in the flow structures of density, pressure, and Lorentz factor shown in 


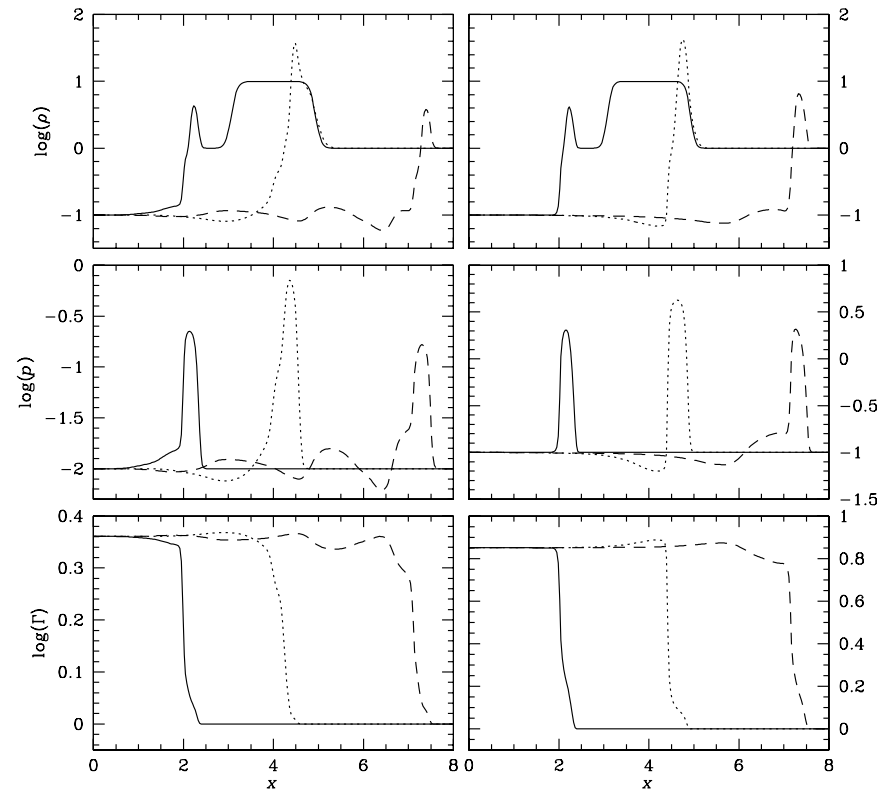

FIG. 2.-Distributions of density, pressure, and Lorentz factor along the beam propagation axis with $y=z=4$ for models M1 (left) and M3 (right) at the same epoch as in Figs. $1 a$ and $1 c$. The solid lines correspond to $t / t_{\mathrm{bc}}=1$, dotted lines represent profiles at $t / t_{\mathrm{bc}}=2.5$, and dashed lines illustrate quantities at $t / t_{\mathrm{bc}}=4$.

Figure 2 at $t / t_{\mathrm{bc}}=4$ (corresponding to the dashed lines) for models M1 and M3, respectively. There are only slight fluctuations in the flow structures at this late stage.

In comparing our simulations with hydrodynamic simulations of nonrelativistic jet-cloud interactions (de Gouveia Dal Pino 1999; Higgins et al. 1999; Wang et al. 2000), we can only note some fairly basic similarities and differences between our relativistic models and the roughly corresponding nonrelativistic models. The lack of good overlap between the $\mathcal{M}_{b}^{R}$ values for the relativistic jets and the standard Mach number for the nonrelativistic jets, as well as differences between cloud size to jet-width ratios considered here and in earlier nonrelativistic work, prevents us from making quantitative comparisons. Relativistic jets interacting with dense clouds certainly do show general morphological features, such as deflections of some gas and bent structures of jets, similar to those found in some of the nonrelativistic jet-cloud interactions. The slower relativistic jet shows a bent structure after interaction, which is similar to that found in nonrelativistic simulations involving "weak" jets, while the faster relativistic jet effectively plows through the clouds. Higher power nonrelativistic jets also can plow through, and apparently completely destroy, clouds. However, some major differences arise because of the larger propagation velocity of the relativistic jets; for example, moderately light $(\eta=0.1)$ relativistic jets are not effectively decelerated and disrupted by the dense $(\chi=100)$ clouds, whereas nonrelativistic jets assaulting clouds of similar density ratios typically are disrupted. Our relativistic jets are rather reaccelerated in either a slightly new or essentially the original direction after their interactions with clouds. The large propagation velocity also suppresses the development of hydrodynamic instabilities in the jets, so that the jets still remain stable and collimated even after the jets smash into much denser clouds. In addition, as discussed in $\S 4.2$, clouds impaled by relativistic jets also appear to survive somewhat better than do those hit by strong nonrelativistic jets.

\subsection{Cloud Evolutions}

Although previous studies of jet interactions with clouds mainly emphasized the dynamical and morphological features of
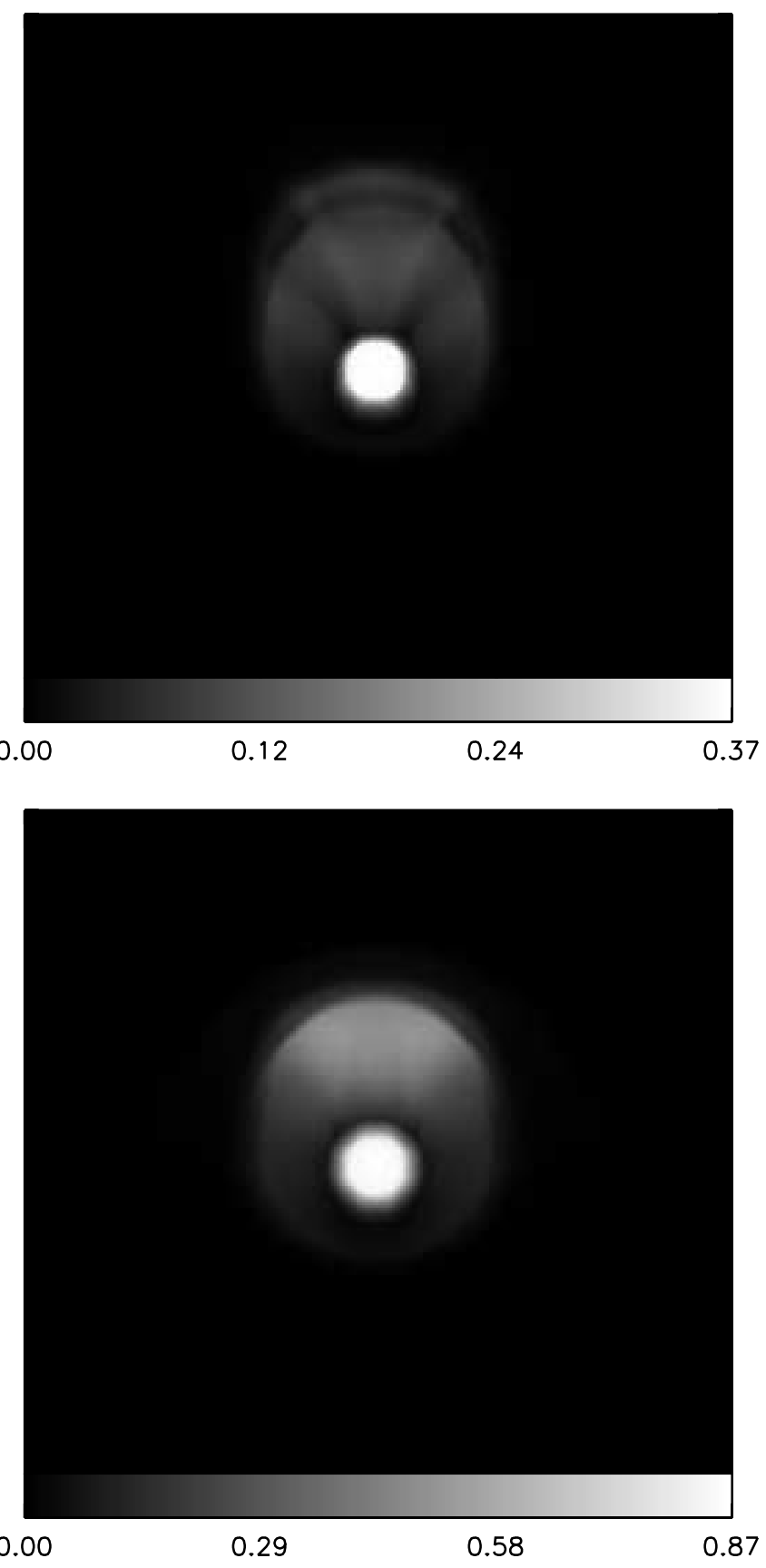

FIG. 3.- Projection images of Lorentz factors at viewing angles of $0^{\circ}$ for models M2 (top) and M4 (bottom) at $t / t_{\mathrm{bc}}=3.5(\mathrm{M} 2)$ and 3 (M4). The image scales are logarithmic and the images are projected on the $y-z$ plane in the threedimensional computational domain.

the jet itself, it is also important to follow the evolution of the clouds during and after the off-axis collisions with relativistic jets. One key reason for investigating the fate of the clouds is that the leftover cloud material is a strong candidate for star formation regions in the vicinity of AGNs (e.g., Rees 1989; Gopal-Krishna \& Wiita 2001). The cloud is expected to undergo a somewhat different evolution in our case compared with the evolution of the clouds struck by the nonrelativistic planar shocks considered in earlier work (e.g., Klein et al. 1994; Xu \& Stone 1995; Mellema et al. 2002; Fragile et al. 2004).

In order to describe the evolution of a cloud quantitatively, we introduce a conserved variable $f$ called a Lagrangian tracer (e.g., Jones et al. 1996), which is updated along with the primitive 
hydrodynamic variables in our simulations. The evolution of the Lagrangian tracer is followed by the conservation equation

$$
\frac{\partial D f}{\partial t}+\frac{\partial}{\partial x_{j}}\left(D f v_{j}\right)=0
$$

Since this conservation equation is almost identical to the mass conservation equation (9), it is separately solved using the same TVD routine adopted for solving the mass conservation equation. Initially the tracer variable is set to unity $\left(f_{c}=1\right)$ inside the cloud while the variable is set to zero $\left(f_{c}=0\right)$ everywhere outside the cloud, so that the density of cloud material is given as $D_{c}=D f_{c}$ (i.e., $\rho_{c}=\rho f_{c}$ ) for a given tracer $f_{c}$ in any zone. Then, the total mass of the cloud is computed by the integration over the entire volume $V$,

$$
m_{c}=\int_{V} \rho_{c} d V
$$

where $d V=d x d y d z$. This enables us to compute the several useful mass-weighted quantities, such as the mean square radius of the cloud and the mean velocity of the cloud,

$$
\begin{gathered}
\left\langle r_{i}^{2}\right\rangle=\frac{1}{m_{c}} \int_{V} \rho_{c} r_{i}^{2} d V, \\
\left\langle v_{i}\right\rangle=\frac{1}{m_{c}} \int_{V} \rho_{c} v_{i} d V .
\end{gathered}
$$

The index $i$ given above represents each spatial component. Another useful mass-weighted quantity is the mean thermal energy inside the cloud $\left\langle e_{\text {th }}\right\rangle$. This is also computed using the same volume integration given above.

We show in Figure 4 the volume-rendering images of cloud density for model M4 at three different times, $t / t_{\mathrm{bc}}=1,3$, and 5 . As a direct consequence of the impact on the cloud by the jet, the cloud develops a cavity in the cloud body as shown in the figure. The cloud cavity continues to grow until the jet completely penetrates the cloud, elongating the cloud material outside the cavity along the bow shock of the jet. Unlike the cases studied earlier, where a cloud interacts with a plane-parallel shock (Klein et al. 1994; Xu \& Stone 1995), the cloud material is not completely destroyed by the impact of the jet. Some cloud mass is carried into the deflected material of the jet, eroding the cloud body, but much of the cloud mass remains in a large, coherent blob for at least a few beam crossing times. This enhancement of the cloud durability is apparently primarily due to the geometric influence of an off-axis collision. Computational resource limits prescribe that we can accurately investigate the clouds for only a few beam crossing times, which is less than the many dynamical times for which it would be desirable to follow their evolutions.

Figure 5 shows for every model the time evolutions of the rms radius of cloud, the mean cloud velocity, and the mean thermal energy of the cloud. In every model the clouds remain in the initial rms radius $\left\langle r_{i}^{2}\right\rangle^{1 / 2}=0.44$ until $t / t_{\mathrm{bc}} \sim 1.5$. When the jet hits the cloud, the cloud is first crushed in the $x$-direction, along which the jet propagates, and then it begins to expand beyond its initial size. The initial compressions in the $y$ - and $z$-directions are very small, and the cloud soon gradually expands in both these transverse directions. By the end of these simulations the rms radii of the clouds have expanded to about 1.5-2 times their initial values.

After $t / t_{\mathrm{bc}} \sim 1.5$ the high pressure inside the cloud generated by the incident jet causes the entire cloud to accelerate. Unsurprisingly, the acceleration is faster in the $x$-direction for the faster jets
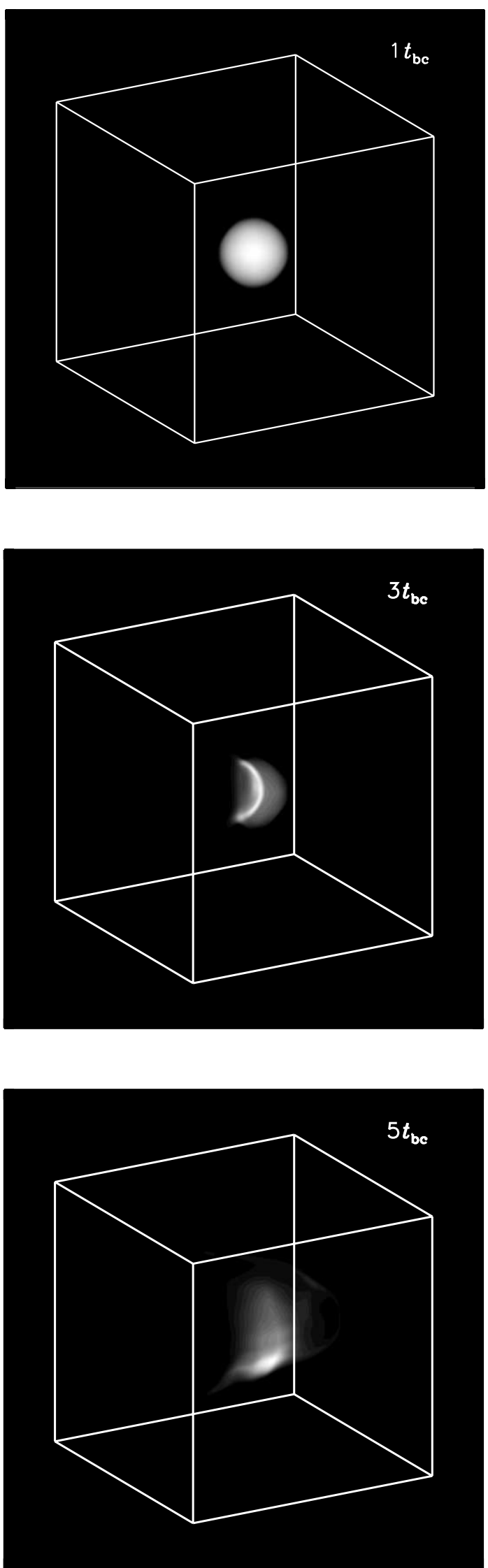

FIG. 4.- Volume-rendering images of cloud density for model M4 at three different times, $t / t_{\mathrm{bc}}=1,3$, and 5 . The image scales are linear and the viewing area is rotated $20^{\circ}$ about the $x$-axis and $30^{\circ}$ about the $z$-axis. Black represents the lowest values that are $\sim 0$ at each epoch and white the highest values that are $\sim 100$ at $t / t_{\mathrm{bc}}=1, \sim 464$ at $t / t_{\mathrm{bc}}=3$, and $\sim 229$ at $t / t_{\mathrm{bc}}=5$. 


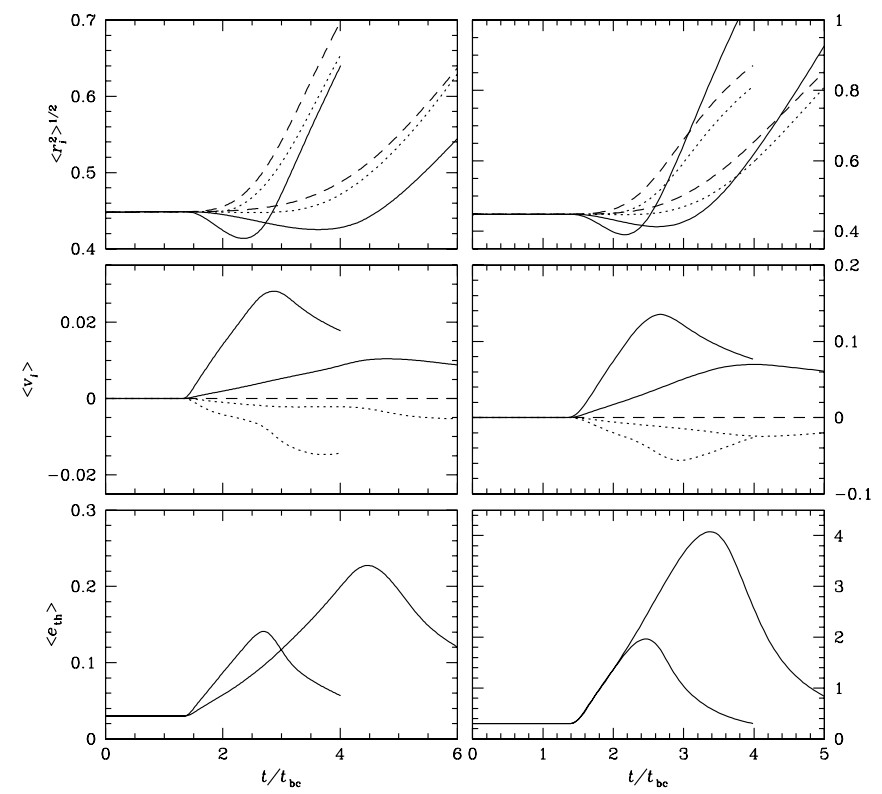

FIG. 5.- Time evolutions of the rms radius of the cloud, the mean cloud velocity, and the mean thermal energy of the cloud for models M1 (curves ending at $t / t_{\mathrm{bc}}=4$ ) and M2 (curves ending at $t / t_{\mathrm{bc}}=6$ ) in the left panels and for models $\mathrm{M} 3$ (curves ending at $t / t_{\mathrm{bc}}=4$ ) and M4 (curves ending at $t / t_{\mathrm{bc}}=5$ ) in the right panels. The solid, dotted, and dashed lines in the rms radius and the mean velocity panels represent the $x$-, $y$-, and $z$-components, respectively.

in models M3 and M4 and for the lighter clouds in models M1 and M3. The mean velocity of the clouds peaks at values between 0.01 and 0.15 in the $x$-direction and 0.005 and 0.05 in the $y$-direction. Note that the mean velocity of the clouds in the $z$-direction is zero because of symmetry in this direction. The maximum velocity of the cloud is always rather modest even if the incident jet has a relativistic speed, although if we had considered less massive clouds they obviously could have been accelerated to higher speeds.

As we expect, the mean thermal energy of the cloud increases while the jet strikes the cloud. The maximum mean thermal energy of the cloud reaches about 5-15 times its initial value, depending on the model. In each model the peaks of the mean thermal energy inside the cloud and the mean velocity of the cloud take place nearly at the same time. Note that at this point the cloud reexpands after the cloud reaches the maximum compression in the $x$-direction.

As mentioned earlier, the jet interaction with the cloud shows that the beam penetrates through the cloud body, which may begin a fragmentation process. A strong shear layer developing at the cloud boundary as a result of the interaction with the jet may lead to Kelvin-Helmholtz instabilities that enable the disrupted cloud body to fragment. So eventually the gas cloud might be broken into small pieces. However, the Kelvin-Helmholtz instability becomes inefficient if the density contrast of two slipping fluids is large or if the flow is supersonic (Chandrasekhar 1961), so we may not see rapid fragmentation in the clouds. Although the fragmentation timescale is difficult to estimate, our simulations show no significant cloud fragmentation by $t / t_{\mathrm{bc}} \sim 4-6$. This indicates that the high density contrast between clouds and beams and supersonic velocity of the clouds induced by the relativistic jets do indeed lower the growth rate of the Kelvin-Helmholtz instabilities.

\subsection{Synchrotron Emission}

Propagating relativistic jets produce nonthermal radio (synchrotron) emission that originates from relativistic high-energy particles accelerated at the shock front. Jones et al. (1999) and
Tregillis et al. (2001) calculated the synchrotron emission in extragalactic jets by explicitly calculating the acceleration of electrons at shocks and following the evolution of magnetic field. However, they assumed nonrelativistic jets, and hence the emissivity needs to be further examined using relativistic jets. To compute the synchrotron emission from relativistic jets, other relativistic hydrodynamic simulations have worked with a simpler approximation (Gómez et al. 1997; Komissarov \& Falle 1997; Mioduszewski et al. 1997; Aloy et al. 2000). Using this same simple model we now calculate the synchrotron emission in our simulations in order to estimate how the relativistic jet interaction with a cloud would appear in emission as a extragalactic radio source. We make the usual assumptions that the jet is optically thin and only the jet material radiates. Thus, in order to separate the jet material from the ambient medium and the cloud, we include an additional tracer variable $f_{b}$ (see $\S 4.2$ ) that is initially set to unity inside the jet $\left(f_{b}=1\right)$ and zero everywhere outside the jet $\left(f_{b}=0\right)$.

The relativistic high-energy electrons responsible for the synchrotron emission are assumed to have a power-law energy distribution. Given the spectral index $\alpha$, the high-energy particle number density $N_{0}$, and the magnetic field intensity $B$, the synchrotron emissivity at frequency $\nu$ is then approximated by the power-law distribution (see e.g., Mioduszewski et al. 1997)

$$
j_{\nu} \propto N_{0} B^{\alpha+1} \nu^{-\alpha}
$$

The high-energy particle number density, $N_{0}$, is assumed to be proportional to the relativistic electron energy density, $u_{e}$, from the integration of the power-law energy distribution over some energy range, and $u_{e}$ is also taken to be proportional to the hydrodynamic pressure. Then we have $N_{0} \propto u_{e} \propto p$. Assuming that there is an equipartition of the magnetic field energy density $u_{B}$ and the relativistic electron energy density $\left(u_{B}=u_{e}\right)$, then $u_{B} \propto p$. This leads to $B \propto u_{B}^{1 / 2} \propto p^{1 / 2}$. Therefore, equation (23) becomes

$$
j_{\nu} \propto p^{(\alpha+3) / 2} \nu^{-\alpha} .
$$

This equation shows that the local thermal pressure approximately reflects the local synchrotron emissivity. We have used $\alpha=0.75$ in our calculation. By integrating the synchrotron emissivity along the line of sight $L$ at a viewing angle $\theta$, we can compute the synchrotron intensity on the surface projected onto the line of sight at the viewing angle

$$
I_{\nu}=\int_{L} \mathcal{D}^{2} j_{\nu} d L,
$$

where the Doppler boosting factor is given by

$$
\mathcal{D}=\frac{1}{\Gamma(1-v \cos \theta)} .
$$

Other relativistic effects, including light aberration and time dilation, have not been included in this calculation, as we assume that these effects are negligible.

Figure 6 shows the synchrotron intensity maps of models M1 and $\mathrm{M} 2$ at the viewing angles $90^{\circ}, 45^{\circ}$, and $0^{\circ}$. These maps are shown at $t / t_{\mathrm{bc}}=2.5$ for M1 and 3.5 for M2 when the jet is colliding with the cloud. The peak intensity in this figure varies with the models and the angles of view. Doppler boosting has a little effect on the emission of the jet at the viewing angle $90^{\circ}$, so that the observed emission is very closely related to the intrinsic emissivity in this case. At smaller viewing angles (e.g., $45^{\circ}$ and $0^{\circ}$ ), however, 

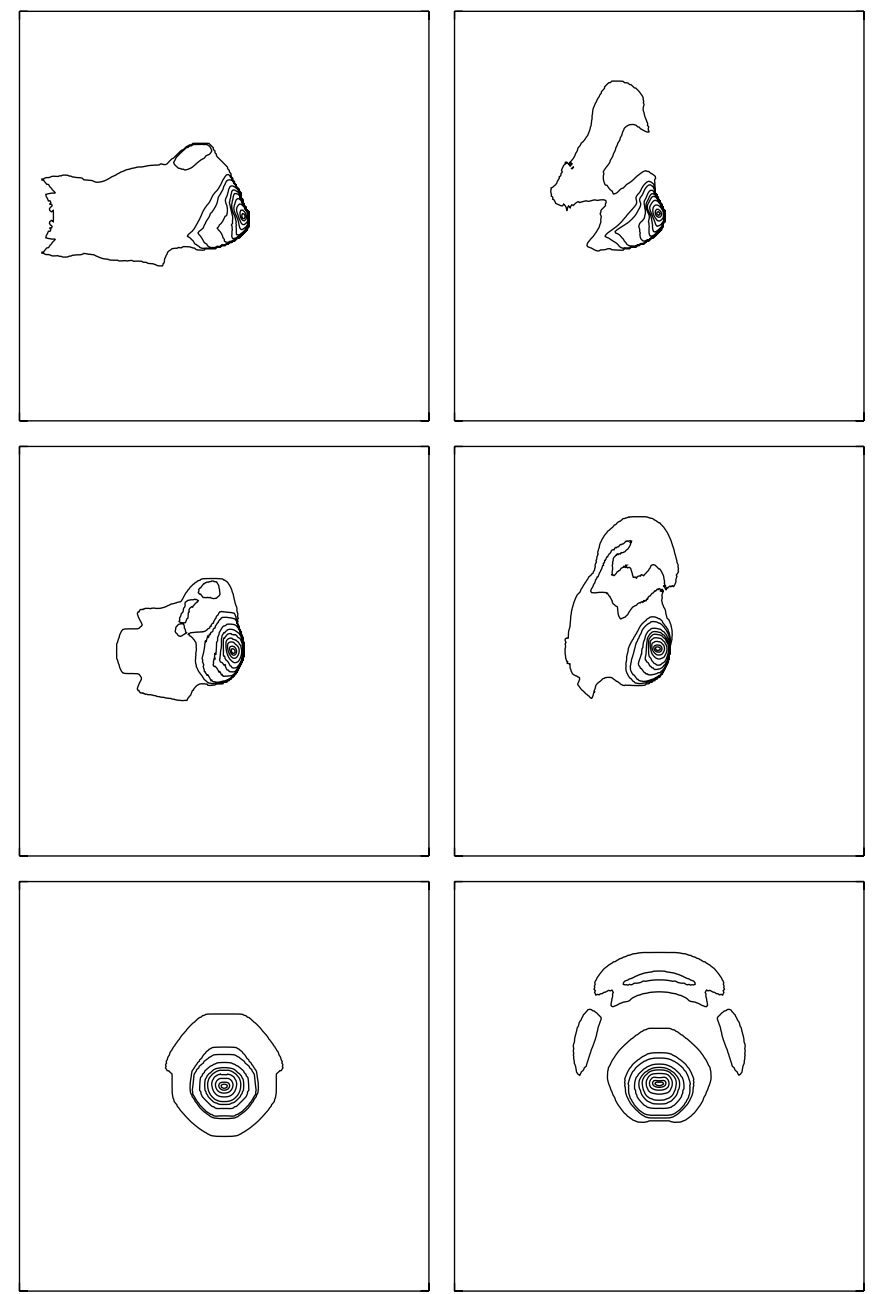

FIG. 6.- Contour maps of synchrotron intensity at the viewing angles of $90^{\circ}$, $45^{\circ}$, and $0^{\circ}$ (top to bottom) for models M1 (left) and M2 (right) at $t / t_{\mathrm{bc}}=2.5$ (M1) and $3.5(\mathrm{M} 2)$. Maximum synchrotron intensities are $0.17\left(90^{\circ}\right), 0.13\left(45^{\circ}\right)$, and $0.27\left(0^{\circ}\right)$ for model M1 and $0.57\left(90^{\circ}\right), 0.35\left(45^{\circ}\right)$, and $0.55\left(0^{\circ}\right)$ for model M2, and the contour levels are $0.1 \%, 0.5 \%, 1 \%, 3 \%, 6 \%, 10 \%, 20 \%, 40 \%, 70 \%$, and $90 \%$ of the maximum synchrotron intensity.

the emission morphology is determined to a large degree by Doppler boosting. The synchrotron emission is dominated by the bright hot spot, which takes the form of the compact emission knot in VLBI radio maps. Although the beam and the deflected material show only weak emission features, there is a faint secondary spot seen from deflected material in model M2.

The time evolution of the total synchrotron intensity for models M1-M4 at the viewing angles of $90^{\circ}, 45^{\circ}$, and $0^{\circ}$ are shown in Figure 7. The total synchrotron intensity computed here is in arbitrary units. There are significant quantitative differences among the models, but the intensity curves show qualitatively the same trends. The total synchrotron intensity is much amplified at smaller viewing angles of $45^{\circ}$ and $0^{\circ}$ because Doppler boosting plays a role in the amplification of the intensity in these cases. As expected, the passage of the jet over a cloud enhances the synchrotron intensity; there are high-amplitude bumps in the intensity curves during the interactions. The total intensity steeply increases at the moment of the impact by the jet, and then gradually increases until the jet crosses over the cloud. This tells us that the compression of the plasma in this region produces higher synchrotron emission in this approximation where it is tied to the pressure. The peak synchrotron intensity occurs shortly after the jet passes through the entire cloud, and
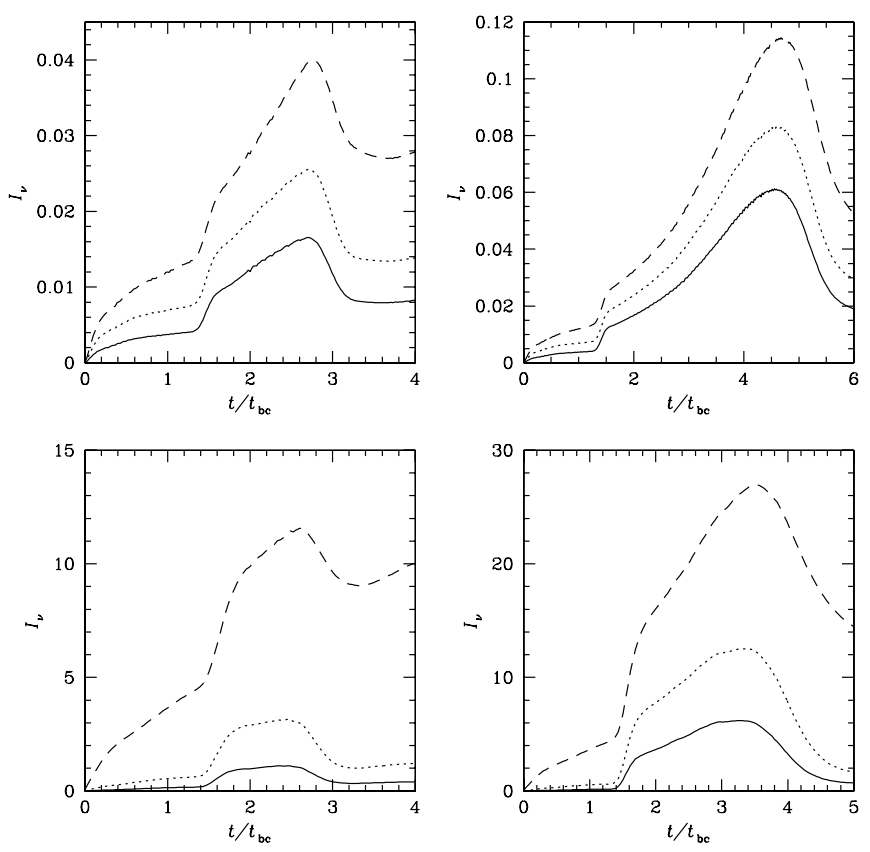

FIG. 7.- Total synchrotron intensity curves for models M1 (top left), M2 (top right), M3 (bottom left), and M4 (bottom right). The solid, dotted, and dashed lines correspond to the viewing angles of $90^{\circ}, 45^{\circ}$, and $0^{\circ}$, respectively.

after that the intensity falls off slowly because the compression is weaker.

Although we have not computed the thermal X-ray emission in detail, we can briefly discuss it. Since the free-free emission (bremsstrahlung) is proportional to $\rho^{2}$, the total X-ray luminosity due to thermal bremsstrahlung is most sensitive to the density of gas, provided that the gas is, or becomes, hot enough to emit X-rays. The relativistic jet itself is not expected to emit thermal X-rays because of its low density, and only in some cases does the synchrotron spectrum extend far enough to produce nonthermal X-ray emission (Harris \& Krawczynski 2006). The dense cloud is very unlikely to start out hot enough to emit X-rays. However, during the jet-cloud interaction the density and pressure of the cloud become so high that the total X-ray emission may be larger inside the cloud than elsewhere, for example, in the bow shock of the jet. An estimate of the increase in the total X-ray luminosity of the cloud is given by $L_{\mathrm{X}} / L_{\mathrm{X}, 0} \simeq\left(\rho_{c} / \rho_{c, 0}\right)^{2}\left(T_{c} / T_{c, 0}\right)^{1 / 2}\left(V_{c} / V_{c, 0}\right)$, where $L_{\mathrm{X}}$ is the total X-ray luminosity of the cloud, $T_{c}$ is the mean cloud temperature, $V_{c}$ is the mean cloud volume, and the subscript 0 represents the initial (preshocked) value. If we simply assume an ideal gas so $T_{c} \propto p_{c} / \rho_{c}$, we have $L_{\mathrm{X}} / L_{\mathrm{X}, 0} \sim\left(\rho_{c} / \rho_{c, 0}\right)^{3 / 2}\left(p_{c} / p_{c, 0}\right)^{1 / 2}\left(V_{c} / V_{c, 0}\right)$, allowing us to estimate the total X-ray luminosity of the cloud with respect to its preshocked X-ray luminosity. In model M4, for example, $\rho_{c} / \rho_{c, 0} \approx 3, p_{c} / p_{c, 0} \approx 12$, and $V_{c} / V_{c, 0} \approx 1$ at $t / t_{\mathrm{bc}}=3$ (as can be roughly estimated from Figs. 4 and 5), so that $L_{\mathrm{X}} / L_{\mathrm{X}, 0} \sim$ 18. Thus, the shocked cloud could possibly be a important source of thermal X-rays depending on the various physical parameters such as the incident jet velocity, the cloud density, and, most importantly, the initial cloud temperature, which is not explicitly specified in our scaled models. However, any significant thermal $\mathrm{X}$-ray luminosity should subside rapidly after the interaction as the cloud is diffusive and quickly attains equilibrium with the postshocked ambient pressure.

\section{SUMMARY AND DISCUSSION}

We have performed three-dimensional relativistic hydrodynamic simulations to study relativistic jet interactions with dense clouds, 
focusing on the influence of special relativistic effects. We have investigated clouds struck by both low and high relativistic beam Mach number jets that have less and more dominant relativistic effects, respectively, and have compared our results to the extent possible with nonrelativistic simulations that have been published previously. We also have studied the evolution of the assaulted clouds and have estimated the synchrotron emission from the relativistic jets interacting with the clouds.

In our models, the partial deflections of the jets due to the interactions with clouds are seen more clearly when denser clouds are involved, and the deflection angle is more strongly influenced by the density contrast of the cloud to the ambient medium than by the beam Mach number of the jet. The streams of deflected gas from the jet induced by the interactions move outward much faster than in nonrelativistic models. If our models can be generalized, this suggests that the relativistic jet-cloud interactions are an effective mechanism of producing at least some of the outflows observed in the vicinity of AGNs (e.g., Emonts et al. 2005; Morganti et al. 2005). After the relativistic jets interact with the dense clouds, we find that the slower relativistic jets can be bent by modest angles and that these bent jets still remain stable and collimated over fairly extended timescales. This trend is similar to the results from nonrelativistic simulations.

The impact of the jet erodes the cloud, but much of the cloud mass survives as a large coherent body rather than being completely destroyed. This enhancement of the cloud durability compared to interactions with planar shocks appears to be primarily due to the geometric influence of an off-axis collision. Compared to head-on collisions, off-axis collisions damage the cloud less, increasing the chance of survival of a large portion of the cloud. Another likely reason for the enhancement of the cloud durability is that the rate of cloud fragmentation through Kelvin-Helmholtz instabilities is lowered, since the relativistic flows reduce the growth rate of the instabilities compared to similar off-axis blows by nonrelativistic jets. This leftover tenacious cloud material could be a candidate for a strong star formation region in the vicinity of AGNs, particularly when the cooling timescales are sufficiently short.

The synchrotron intensity "maps" show that at the jet impact on a cloud, the synchrotron emission comes dominantly from a bright hot spot that could correspond to the form of the compact emission knots seen in many VLBI radio maps. Although the emission from the deflected jet material is relatively weak, there is a secondary synchrotron spot visible from this deflected material. This emission feature may represent some of the distorted emission seen in many VLBI radio maps. The passage of the jet over a cloud significantly enhances the total synchrotron intensity of the jet. We find that the synchrotron emission is steeply enhanced shortly after the jet hits the cloud, but the emission peaks right before the jet passes through the cloud. The next big step in performing these calculations would be to include magnetic fields. Such relativistic magnetohydrodynamical simulations would allow for better estimates of synchrotron emission and would allow the examination of the polarization of emission arising from more complicated shock structures. Such polarization structures could be a useful diagnostic of the dynamics.

Although most astrophysical simulations based on relativistic hydrodynamics, including this study, have assumed the ideal EOS, it is well known that the ideal EOS is correct only if the gas is assumed to be entirely nonrelativistic $(\gamma=5 / 3)$ or ultrarelativistic $(\gamma=4 / 3)$. If a local transition between nonrelativistic gas and relativistic gas is involved, the ideal EOS will produce incorrect results in that regime. Recently, Ryu et al. (2006) have studied this issue of the EOS in numerical relativistic hydrodynamics and propose a new EOS that is simple and yet approximates closely the EOS of a perfect gas in the relativistic regime, having an accuracy in enthalpy better than $0.8 \%$. Future numerical simulations using this new EOS should produce even better results concerning the problem of relativistic jet interactions with clouds.

We thank the anonymous referee for several suggestions that improved the presentation in this paper. The simulations were performed on the Linux Biocluster at Georgia State University, and we are grateful for the allocation of substantial time on this cluster. E. C. and P. J. W. have been supported in part by a subcontract to GSU from NSF grant AST 0507529 to the University of Washington. D. R. has been supported in part by the KOSEF grant R01-2004-000-10005-0.

\section{REFERENCES}

Aloy, M. A., Gómez, J. L., Ibáñez, J. M., Martí, J. M., \& Müller, E. 2000, ApJ, $528, \mathrm{~L} 85$

Aloy, M. A., Ibáñez, J. M., Martí, J. M., Gómez, J. L., \& Müller, E. 1999, ApJ, 523, L125

Chandrasekhar, S. 1961, Hydrodynamic and Hydromagnetic Stability (Oxford: Clarendon Press)

Choi, E., \& Ryu, D. 2005, NewA, 11, 116

de Gouveia Dal Pino, E. M. 1999, ApJ, 526, 862

Duncan, G. C., \& Hughes, P. A. 1994, ApJ, 436, L119

Emonts, B. H. C., Morganti, R., Tadhunter, C. N., Oosterloo, T. A., Holt, J., \& van der Hulst, J. M. 2005, MNRAS, 362, 931

Fanaroff, B. L., \& Riley, J. M. 1974, MNRAS, 167, 31P

Fragile, P. C., Murray, S. D., Anninos, P., \& van Breugel, W. 2004, ApJ, 604, 74

Gómez, J. L., Martí, J. M., Marscher, A. P., Ibáñez, J. M., \& Alberdi, A. 1997, ApJ, 482, L33

Gopal-Krishna, \& Wiita, P. J. 2001, ApJ, 560, L115

Harris, D. E., \& Krawczynski, H. 2006, ARA\&A, 44, 463

Harten, A. 1983, J. Comput. Phys., 49, 357

Higgins, S. W., O’Brien, T. J., \& Dunlop, J. S. 1999, MNRAS, 309, 273

Hughes, P. A., Miller, M. A., \& Duncan, G. C. 2002, ApJ, 572, 713

Jones, T. W., Ryu, D., \& Engel, A. 1999, ApJ, 512, 105

Jones, T. W., Ryu, D., \& Tregillis, I. L. 1996, ApJ, 473, 365

Klein, R. I., McKee, C. F., \& Colella, P. 1994, ApJ, 420, 213

Komissarov, S. S., \& Falle, S. A. E. G. 1997, MNRAS, 288, 833 1998, MNRAS, 297, 1087

Königl, A. 1980, Phys. Fluids, 23, 1083

Landau, L. D., \& Lifshitz, E. M. 1959, Fluid Mechanics (London: Pergamon Press)

Martí, J. M., Müller, E., Font, J. A., Ibáñez, J. M., \& Marquina, A. 1997, ApJ, 479,151

Martí, J. M., Müller, E., \& Ibáñez, J. M. 1994, A\&A, 281, L9

Mellema, G., Kurk, J. D., \& Röttgering, H. J. A. 2002, A\&A, 395, L13

Mioduszewski, A. J., Hughes, P. A., \& Duncan, G. C. 1997, ApJ, 476, 649

Mizuta, A., Yamada, S., \& Takabe, H. 2004, ApJ, 606, 804

Morganti, R., Oosterloo, T. A., Tadhunter, C. N., van Moorsel, G., \& Emonts, B. 2005, A\&A, 439, 521

Mundell, C. G., Wrobel, J. M., Pedlar, A., \& Gallimore, J. F. 2003, ApJ, 583, 192 Poludnenko, A. Y., Frank, A., \& Blackman, E. G. 2002, ApJ, 576, 832

Rees, M. J. 1989, MNRAS, 239, 1P

Rosen, A., Hughes, P. A., Duncan, G. C., \& Hardee, P. E. 1999, ApJ, 516, 729

Ryu, D., Chattopadhyay, I., \& Choi, E. 2006, ApJS, 166, 410

Saxton, C. J., Bicknell, G. V., Sutherland, R. S., \& Midgley, S. 2005, MNRAS, 359,781

Tregillis, I. L., Jones, T. W., \& Ryu, D. 2001, ApJ, 557, 475

van Putten, M. H. P. M. 1993, ApJ, 408, L21

Wang, Z., Wiita, P. J., \& Hooda, J. S. 2000, ApJ, 534, 201

Wiita, P. J., \& Norman, M. L. 1992, ApJ, 385, 478

Wiita, P. J., Rosen, A., \& Norman, M. L. 1990, ApJ, 350, 545

Wilson, J. R., \& Mathews, G. J. 2003, Relativistic Numerical Hydrodynamics

(Cambridge: Cambridge Univ. Press)

Xu, J., \& Stone, J. M. 1995, ApJ, 454, 172

Zensus, J. A. 1997, ARA\&A, 35, 607 